\title{
1 A Water-based Neutron Detector as a Well Multiplicity Counter
}

2

3 S. Dazeley ${ }^{a^{*}}$, A. Asghari ${ }^{\mathrm{b}}$, A. Bernstein ${ }^{\mathrm{a}}$, N. S. Bowden ${ }^{\mathrm{a}}$, V. Mozin ${ }^{\mathrm{a}}$

$4 \quad{ }^{a}$ Lawrence Livermore National Laboratory, Livermore, CA 94550, USA

$5 \quad{ }^{b}$ Department of Nuclear Engineering, University of California, Berkeley, CA 94720, USA

6

7 Abstract

8 We report the performance characteristics of a water-based neutron detecting multiplicity counter for the

9 non-destructive assay of fissile sources. This technique could replace or supplement existing ${ }^{3} \mathrm{He}$-based

10 multiplicity counters. The counter is a $1.02 \mathrm{~m}^{3}$ tank containing pure deionized water doped with $0.5 \%$

$11 \mathrm{GdCl}_{3}$. It has highly reflective walls and eight 10-inch PMTs mounted at the top. An unshielded source

12 well of $19 \mathrm{~cm}$ diameter, mounted at the top and center, extends $73 \mathrm{~cm}$ down into the detector. The counter

13 was evaluated using low intensity ${ }^{252} \mathrm{C}$ and ${ }^{60} \mathrm{Co}$ sources, and a fast pulsing LED to simulate higher

14 intensity backgrounds. At low gamma ray intensities ( $200 \mathrm{kBq}$ or less) we report an absolute neutron

15 detection efficiency of $28 \%$ and a ${ }^{60} \mathrm{Co}$ rejection/suppression factor of $\sim 10^{8}$ to 1 . For sources with high

16 gamma ray intensities, the neutron efficiency was $22 \% \pm 1 \%$ up to a ${ }^{60}$ Co equivalent activity of $4 \mathrm{MBq}$.

17 The detector background event rate, primarily due to muons and other cosmogenic particles, was found to

18 be stable over a period of almost three months. The minimum detectable neutron source intensity above

19 background was 3.1 neutrons/second, assuming a one-hour data acquisition.

20

21 Keywords: Water Cherenkov, neutron detector, multiplicity well counter, spontaneous fission, spent fuel,

22 gadolinium

23

\section{1. Introduction}

25 In recent years the severe shortage of ${ }^{3} \mathrm{He}$ has been a great concern for organizations involved in nuclear

26 security ([1],[2],[3]). ${ }^{3} \mathrm{He}$-based ionization tubes are uniquely suited for neutron detection: they are safe and

27 non-cryogenic, exploit the high neutron capture cross section of ${ }^{3} \mathrm{He}$, and have excellent gamma ray

* Corresponding author: Lawrence Livermore National Laboratory, 7000 East Ave, L-211, Livermore, CA, 94550. Ph: 925423 4792, Email address: dazeley2@llnl.gov 
1 rejection. Detector configurations comprising tightly packed arrays of ${ }^{3} \mathrm{He}$ tubes within a moderating

2 material such as polyethylene are highly efficient and can be used to detect multiple neutrons arising from

3 single fissions, and hence measure the fissile content of samples of special nuclear material (e.g. [4].[5])

$4 \quad{ }^{3} \mathrm{He}$-based well counting systems range in efficiency from $10 \%$ to $50 \%$, depending on how tightly tubes are

5 packed and the ${ }^{3} \mathrm{He}$ gas density. Highly efficient and large systems, however, require the use of a large

6 fraction of the yearly supply of ${ }^{3} \mathrm{He}$ and are prohibitively expensive. In recent years the number of

7 competing neutron detection techniques has proliferated in response to the ${ }^{3} \mathrm{He}$ shortage, but many are not

8 yet ready for widespread use. Boron-based systems such as $\mathrm{BF}_{3}$ and ${ }^{10} \mathrm{~B}$ tubes/planes present toxicity

9 concerns and/or are relatively inefficient. Scintillator-based solutions often rely on differences in signal

10 pulse shape to discriminate against gamma rays, placing severe limits on the event rate that can be tolerated

11 before pileup issues dominate. Germanium or silicon-based detectors are small, reducing their overall

12 efficiency. Due to deployment of large-volume neutron detectors at US borders and increased demand for

13 medical imaging, US federal ${ }^{3} \mathrm{He}$ reserves have decreased from 220,000 liters in 2001 to 50,000 liters in

142010 [2]. The cost of ${ }^{3} \mathrm{He}$ has increased from $\$ 45-\$ 85$ per liter prior to the shortage to $\$ 600-\$ 1000$ per liter

15 in 2011. Since the ${ }^{3} \mathrm{He}$ shortage is projected to continue for the foreseeable future, alternative techniques

16 are needed.

17

18 Coincidence counting of neutrons is an effective way to non-destructively determine the amount of fissile

19 material within a sample of special nuclear material (SNM) [6]. The technique is to measure pairs of

20 neutrons correlated in time from single fission events. For many fissile source configurations, multiplicity

21 counting is a more powerful and general technique. However, it requires detection of three or more

22 neutrons from a single fission event. Since the efficiency for detection of $\mathrm{n}$ coincident neutrons scales as

23 the nth power of the efficiency for one neutron, the single neutron detection efficiency quickly becomes the

24 most important criterion for evaluation of new technologies. Of the options available, despite their toxicity,

$25 \mathrm{BF}_{3}$ gas detectors have been considered the most viable alternative to ${ }^{3} \mathrm{He}$ for safeguards applications,

26 because of the stringent efficiency requirements [7]. 
1 In evaluating the efficacy of a neutron coincidence counting technique, the safeguards figure-of-merit

2 (FOM) is a standard metric ([7],[8]):

3

$4 \quad F O M=\frac{\varepsilon}{\sqrt{\tau}}$.

5

$6 \varepsilon$ is the single neutron detection efficiency and $\tau$ the mean thermal neutron capture time (often referred to as

7 the die-away time). A second important performance criterion is the ability of the detector to maintain high

8 neutron detection efficiency and low dead time in the presence of a high gamma ray dose rate. Dose rates

9 at the detector face may be as high as $500 \mathrm{mR} / \mathrm{h}$ for spent nuclear fuel sources [9]. High gamma ray dose

10 rates, however, are only significant in the context of a water Cherenkov detector if the gamma rays are of

11 sufficiently high energy. Extreme levels of gamma ray emission from ${ }^{137} \mathrm{Cs}$ or ${ }^{241} \mathrm{Am}$ are likely to produce

12 almost no water Cherenkov response whatsoever, as we demonstrate below.

13

14 In recent years we have studied a number of water-based detectors for the purpose of detecting neutrons

15 ([10],[11],[12]), achieving neutron efficiencies in the $20 \%$ to $30 \%$ range depending on the materials used

16 and the application. We present here an investigation into the utility of using a water-based neutron

17 detector for the purpose of non-destructive assay (NDA) of special nuclear material.

18

19 2. The Water-Based Well Counter

20

21 The active volume of the Water-Based Well Counter (WBWC) comprises $1.02 \mathrm{~m}^{3}$ of pure $18 \mathrm{MOhm}$

22 deionized water doped with $0.5 \%$ gadolinium-chloride $\left(\mathrm{GdCl}_{3}\right)$, contained within a stainless steel tank

23 (121.9 $\mathrm{cm} \times 91.4 \mathrm{~cm} \times 119.4 \mathrm{~cm})$. To protect the doped water from the corrosive effects of chlorine on

24 stainless steel [13], the inside of the tank was coated with a baked on layer of Teflon. Figure 1 shows a

25 schematic and picture of the detector. There are eight waterproof Hamamatsu R7081 10-inch PMTs

26 mounted at the top of the detector looking down into the water volume. The water level is filled to half

27 way up the PMT bulbs so that they are approximately neutrally buoyant. All of the PMT supports were

28 constructed from clear acrylic or reflective white polypropylene, relatively inert polymers that do not react 
with deionized water, to maximize the transmission and/or reflection of photons in the detector. Also

2 mounted from the top in the center is a $19 \mathrm{~cm}$ diameter well, or source cavity, that extends $73 \mathrm{~cm}$ down into

3 the tank (approximately $45 \mathrm{~cm}$ into the water). The well accommodates square samples as large as $15 \times 15$

$4 \mathrm{~cm}^{2}$. In order to efficiently transport Cherenkov photons to the PMTs the walls of the tank were also

5 coated with a $1.0 \mathrm{~mm}$ reflective layer of $\mathrm{GORE}^{\circledR} \mathrm{DRP}^{\circledR}$, - a Teflon-based highly reflective material (> 99\%

6 in the blue and near UV).

7

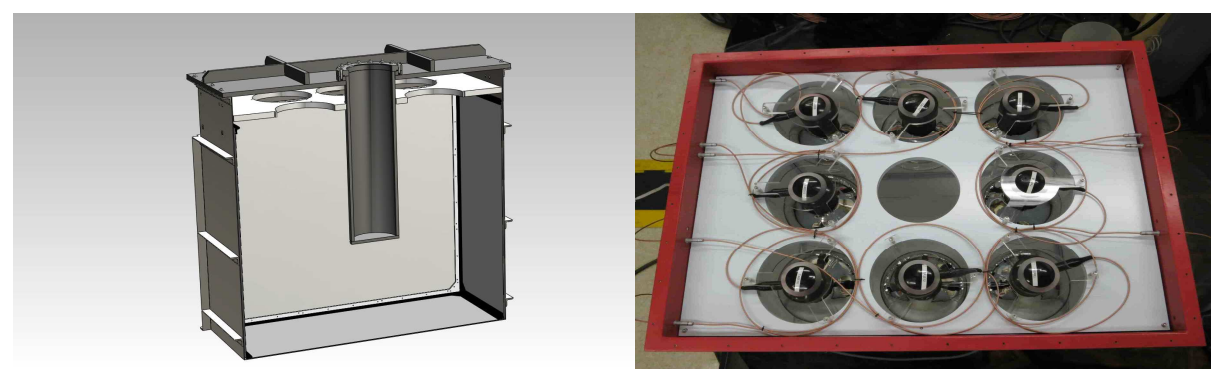

Figure 1: A Schematic (left) of the detector showing a cut away of the $73 \mathrm{~cm}$ deep source deployment well/cavity and PMT placement (PMTs not shown). To the right is the finished detector immediately after PMT placement inside and prior to the installation of the lid and well.

Signals from each of the eight PMTs were sent into a CAEN V975 fast amplifier where they were amplified and split, with one signal sent to a CAEN V814 discriminator and the other to a Struck SIS3320 waveform digitizer (WFD). The trigger was generated by a CAEN V1495 FPGA from the simultaneous arrival of any three discriminator signals. Once a trigger is issued, the WFD can either record full microsecond ( $\mu \mathrm{s})$ long waveforms with a 5 nanosecond (ns) sampling interval, or digitize a set of independently integrated waveform sections for each PMT.

The PMT gains were set relatively high $\left(\sim 10^{7}\right)$ to resolve single photoelectron peaks, enabling easy gain calibration via a green LED permanently mounted inside the detector.

\section{Characteristic Response to Neutrons and Gamma Rays}

Spontaneous fission sources, such as ${ }^{252} \mathrm{Cf}$, emit coincident gamma rays and neutrons with every fission, which can result in a set of correlated events in the detector. If the fission gamma rays are of sufficient 
1 energy $(\sim 1 \mathrm{MeV})$ and multiplicity, they may produce an instantaneous response in the WBWC. Neutrons

2 are efficiently moderated in the active water volume and preferentially capture on gadolinium. The large

3 gadolinium capture cross section results in a short mean capture time of $16 \mu \mathrm{s}$. When multiple gamma rays

4 and neutrons are produced simultaneously, the result is a sequence of correlated events, beginning with

5 either Cherenkov light from above-threshold gamma ray(s), or with a neutron capture (if the prompt

6 gamma rays failed to trigger the detector), and followed by delayed neutron captures. In any given

7 correlated sequence, events occurring after the first event are more likely to be neutron captures.

8 Uncorrelated event sequences may also arise, from the random arrivals of background gamma rays, or from

9 two or more different source fissions.

\section{0}

11 Figure 2 shows the distribution of time intervals between successive events from a one-hour calibration run

12 with a $1.0 \mu \mathrm{Ci}^{252} \mathrm{Cf}$ fission source placed at the base of the source cavity. The inter-event time distribution

13 has two exponential components - a fast decaying correlated component with mean inter-event time 12.3

$14 \mu \mathrm{s}$, and an uncorrelated component with mean inter-event time of $395 \mu$ s. The short time constant

15 exponential is associated with the correlated neutron bursts of interest. This is shown in Figure 3, where

16 events with small inter-event times have a spectral shape enhanced at higher energies by the excess of

17 neutron captures. The underlying uncorrelated component, however, has the same spectral shape as events

18 with long inter-event times. We subtract this component using the normalization provided by the

19 exponential fit of the uncorrelated inter-event time distribution. Figure 3 illustrates this statistical

20 subtraction, which results in a spectrum that corresponds to the WBWC response to neutron captures on

21 gadolinium and hydrogen. 


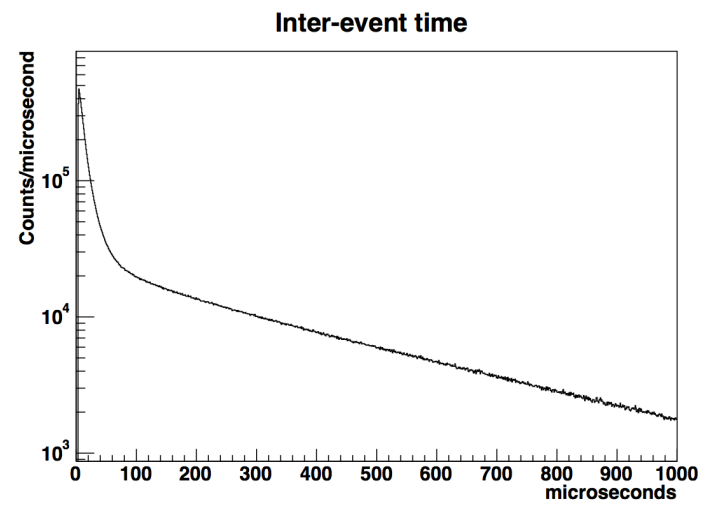

1

2 Figure 2: A plot of the inter-event time distribution for a one-hour measurement of a ${ }^{252} \mathrm{Cf}$ source in the source 3 well/cavity. The distribution is well fit by a double exponential function - indicating a correlated and non-correlated 4 component. The correlated component, at small inter-event times, is due to the thermalization and capture of multiple 5 simultaneously emitted neutrons in the detector. Its exponential has a time constant consistent with a mean inter-event 6 time of $12.3 \mu \mathrm{s}$.

Detector Response (Cf-252)

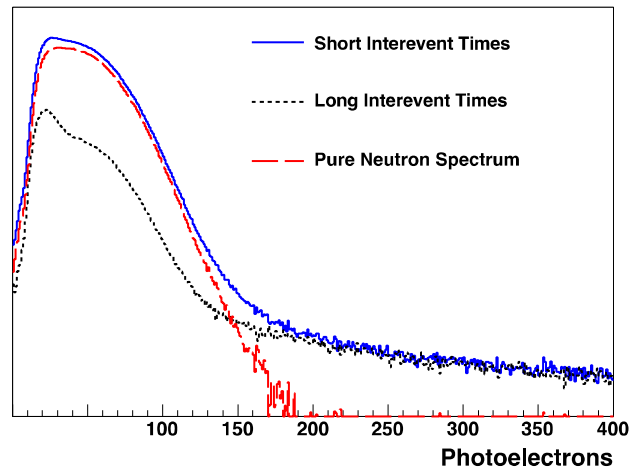

$\mathbf{7}$

11
Figure 3: A comparison of the charge spectrum for event pairs with short inter-event times and long inter-event times. In both datasets the number of uncorrelated events is the same. The statistical subtraction of the two, which gives the background free spectrum of neutron capture events, is also shown.

220 kBq Co-60 Detector Response

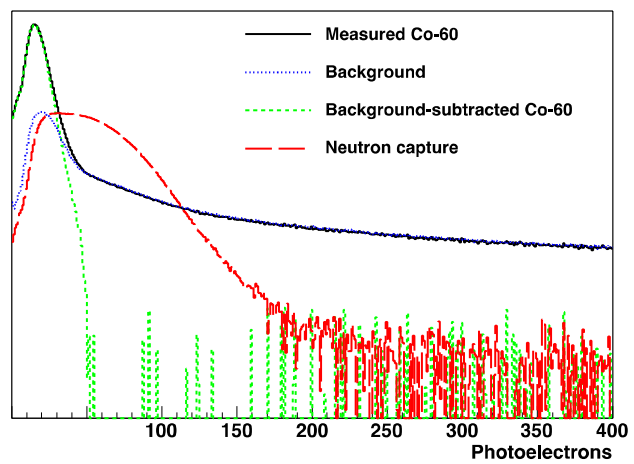

Figure 4: The detector response of a $220 \mathrm{kBq}{ }^{60} \mathrm{Co}$ source for a one-hour data acquisition. Also shown is a one-hour background run, and the background subtracted ${ }^{60} \mathrm{Co}$ response. Shown for comparison is the "pure" neutron capture spectral response that was generated in Figure 3. 
1 Figure 4 shows the spectral response of the detector to a $220 \mathrm{kBq}(5.9 \mu \mathrm{Ci}){ }^{60} \mathrm{Co}$ source positioned inside

2 the well for a one hour data acquisition, compared to a one-hour no source background run. The ${ }^{60} \mathrm{Co}$

3 source was used as a proxy for any kind of source that emits a low intensity gamma ray background at an

4 energy of approximately 1 to $2 \mathrm{MeV}$. Also shown for comparison is the "pure" neutron capture spectrum

5 generated in Figure 3. By requiring a selection criterion of at least 50 photoelectrons it is possible to

6 remove all evidence of the ${ }^{60} \mathrm{Co}$ background while maintaining high neutron detection efficiency.

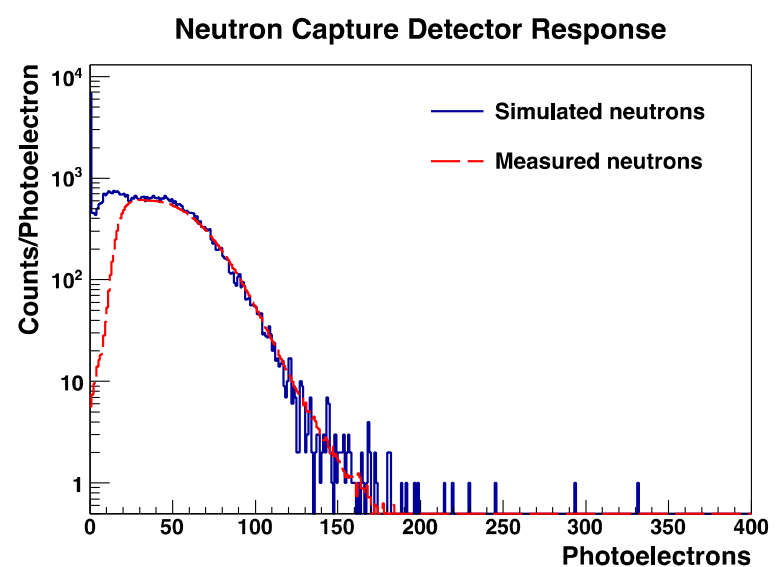

11 average PMT quantum efficiency. In our model, the average wall reflectivity was set to $93 \%$ - this was a

18 simplification of the real detector, which comprised side and base walls, covered in GORE DRP (99\%), and

19 polypropylene between the PMTs at the top and around the edges of the detector, assumed to be

20 approximately $80 \%$ reflective. The water attenuation length was modeled by a function that reaches a

21 maximum of about 35 meters. The functional shape was taken from a measurement at Super-Kamiokande

22 [17]. The PMT quantum efficiency $(\mathrm{QE})$ was modeled using data supplied by the manufacturer. To 
1 account for the fact that the PMTs were not magnetically shielded the QE was multiplied by a factor of

20.85.

3

4

5

7

10 between 50 and 200 photoelectrons. The efficiency was also calculated simply from the nominal ${ }^{252} \mathrm{Cf}$

11 source activity, last measured in October 2007 at $185 \mathrm{kBq}$. The uncertainty associated with this activity is

12 unknown, but typically manufacturers quote approximately $10 \%$ uncertainty for check sources of this type.

13 Our measurements were made approximately 6 years (2.26 half lives) later, implying source intensity of 38

$14 \pm 4 \mathrm{kBq}(1.0 \mu \mathrm{Ci})$. The WBWC detected 1230 neutrons out of a possible 4400 per second, at an efficiency

15 of $28 \pm 3 \%$, in excellent agreement with the predicted value from simulation.

16

17 Since ${ }^{137}$ Cs normally contributes a significant fraction of the radioactivity of spent fuel, the detector

18 response to background $662 \mathrm{keV}$ gamma rays from ${ }^{137} \mathrm{Cs}$ was also investigated. The spectrum from a one-

19 hour run using a $338 \mathrm{kBq}(9.2 \mu \mathrm{Ci}){ }^{137} \mathrm{Cs}$ source was generated and compared with a no source run of the

20 same duration. Both spectra were almost identical due to the inherent lack of Cherenkov light that can be

21 produced from the Compton scattering of $662 \mathrm{keV}$ gamma rays - the scattered electrons are only

22 marginally above the Cherenkov threshold in water. The lack of response from these low energy gamma

23 rays is an attractive feature of the Cherenkov effect in this context. The ${ }^{137}$ Cs source produced no events in

24 the detector greater than 25 photoelectrons. The integrated contribution to the raw trigger rate was only a

$2510 \mathrm{~Hz}$ increase.

\section{6}

27 In Figure 4 the background spectrum was obtained with no radioactive sources present. Over the energy

28 range we use to select neutron capture events (50 to 200 photoelectrons), the background event rate was

29 approximately $155 \mathrm{~Hz}$. Both the background rate and the spectral response of the detector have remained 
remarkably constant over the full period of data taking. Over that time period the neutron background event rate dropped only slightly, from $155( \pm 0.2)$ to $154.8( \pm 0.2) \mathrm{Hz}$, again indicating that the water attenuation

3 has not suffered long-term variations significant enough to negatively impact detector performance. So

4 long as the background rate is stable, the WBWC can be sensitive to increases in neutron emission rate as

5 small as $0.88 \mathrm{~Hz}$ (to $3 \sigma$ ), allowing for low rates of gamma ray emission, such as from the ${ }^{137} \mathrm{Cs}$ and ${ }^{60} \mathrm{Co}$

6 sources used here. The minimum detectable source activity is therefore 3.1 neutrons per second assuming $28 \%$ absolute detector efficiency.

8

9
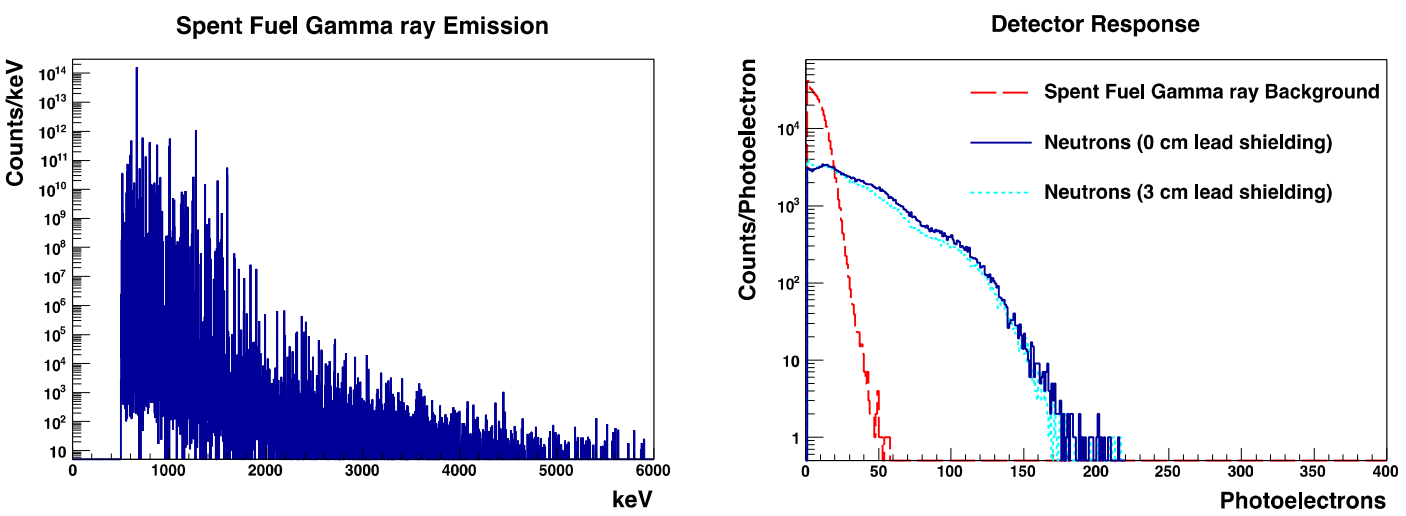

Figure 6: The gamma ray emission spectrum from a single $20 \mathrm{~cm}$ spent fuel pin with burnup of $30 \mathrm{GWd} / \mathrm{ton}$ and cooling time of 20 years (left). The simulated detector response to this source with $3 \mathrm{~cm}$ of lead shielding inside the source cavity is shown at right. The neutron capture detector response for an arbitrary number of neutrons is also shown for comparison, with and without $3 \mathrm{~cm}$ of lead shielding.

15

16

17

18

19

20

21

22

To summarize, water Cherenkov detectors can provide sufficient energy resolution to discriminate between neutron capture events and low energy gamma ray events. By selecting neutrons on the basis of their detector spectral response alone, the WBWC can achieve an absolute neutron efficiency of $28 \%$ while removing very close to $100 \%$ of the background from a ${ }^{60} \mathrm{Co}$ source (which we use here as a proxy for gamma ray background associated with a fission source). We include all the neutrons emitted by the fission source in our efficiency estimate, not simply the neutrons incident on the detector walls. The ${ }^{60} \mathrm{Co}$ rejection factor at this efficiency is approximately $10^{8}$ to 1 . Remarkably, this is competitive with ${ }^{3} \mathrm{He}$-based detection. Discrimination against lower energy gamma rays from a ${ }^{137} \mathrm{Cs}$ source is even better.

To demonstrate the utility of the WBWC in the presence of very high gamma ray backgrounds, such as spent fuel measurements, three factors remain to be studied. Firstly, no gamma ray shielding was used to 
1 reduce detector susceptibility to high intensity gamma ray sources. Low energy gamma ray susceptibility

2 can be expected to be reduced further by such a shielding layer, with perhaps some loss of neutron

3 efficiency. Secondly, unlike common ${ }^{3} \mathrm{He}$-based detectors of this type, our detector is monolithic.

4 Segmentation may be exploited in the future to further reduce the detector's overall susceptibility to high

5 gamma ray source rates. Since each segment would only need to contend with a fraction of the background

6 rate of a monolithic detector. Thirdly, unlike our ${ }^{60} \mathrm{Co}$ spent fuel proxy, a real spent fuel sample would

7 produce some high-energy gamma rays $(>\sim 5 \mathrm{MeV})$. To predict the detector response to a real world spent

8 fuel sample we used a gamma ray emission model from the NGSI spent fuel library number 2,

9 (http://www.lanl.gov/orgs/ndo/n4/documents/sfl2a.zip), and modeled the response of the WBWC with the

10 tuned GEANT4 detector simulation. In Figure 6 (left) we show the gamma ray emission spectrum used as

11 input to our model. This represents an integrated 900 -second gamma ray source spectrum from a $20 \mathrm{~cm}$

12 long fuel pin subjected to a burnup of $30 \mathrm{GWd} /$ ton and a cooling time of 20 years. Note that the most

13 intense emission line, by two orders of magnitude, is ${ }^{137} \mathrm{Cs}$ at $662 \mathrm{keV}$, and the range of intensities extend

14 over 14 orders of magnitude. The modeled detector response to these emissions is shown in Figure 6

15 (right), assuming $3 \mathrm{~cm}$ of lead shielding inside the source cavity. For comparison the neutron capture

16 spectral response for an arbitrary number of neutron captures is also shown, with and without $3 \mathrm{~cm}$ lead

17 shielding. The simulation suggests that the $3 \mathrm{~cm}$ of lead shield would only produce a small effect on the

18 neutron efficiency. The effect of pileup resulting from an intense flux of low energy gamma rays is

19 investigated in the next section. However, ignoring these effects for now, the spent fuel gamma ray rate

20 from our simulated spent fuel source that passes the neutron selection cut (50-200 photoelectrons) was less

21 than $10 \mathrm{~Hz}$. 


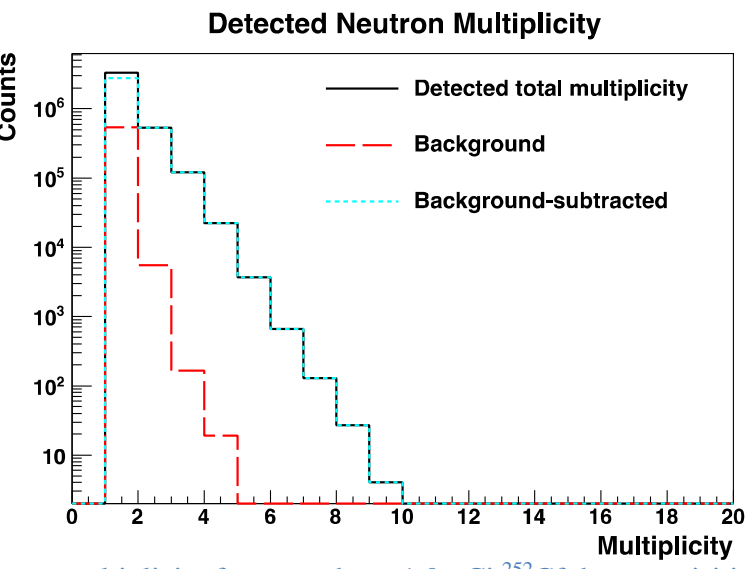

$\mathbf{1}$

Figure 7: The detected neutron multiplicity for a one-hour $1.0 \mu \mathrm{Ci}{ }^{252} \mathrm{Cf}$ data acquisition compared with background.
The background subtracted ${ }^{252} \mathrm{Cf}$ multiplicity is also shown. See text for definitions.

4 In all of the following the above neutron selection cut is applied by default, Figure 7 shows the detected

5 multiplicity from a one-hour data acquisition with the $1.0 \mu \mathrm{Ci}^{252} \mathrm{Cf}$ source (black), compared to a one-hour

6 no source background run (light blue). The background-subtracted multiplicity is also shown in dark blue.

7 We group any two neutron-like events into the same multiplicity set if they fall within $50 \mu$ s of each other.

8

9

\section{4. Detector Performance in the Presence of Intense Gamma Ray Activity}

\section{1}

12 Until now we have only considered neutron/gamma ray discrimination on an event-by-event basis, at rates

13 consistent with little to no pileup. Here we consider performance effects that may result from very high

14 rates of low energy gamma ray emission, such as from a spent fuel source. Even if gamma ray background

15 is not energetic enough to trigger the detector, extremely high rates can produce an almost continuous level

16 of background light inside the detector, which may impact the efficiency and/or spectral response of the

17 PMTs at higher energies. In anticipation of these effects we first optimized the PMT multiplicity trigger by

18 comparing the measured detector response to the neutron simulation. The efficiency was calculated as a

19 function of photoelectrons detected for each of the possible PMT trigger multiplicities $(1 \rightarrow 8)$. A trigger

20 multiplicity of three producing the fastest rising efficiency curve was employed as the default trigger in all

21 subsequent work.

22 


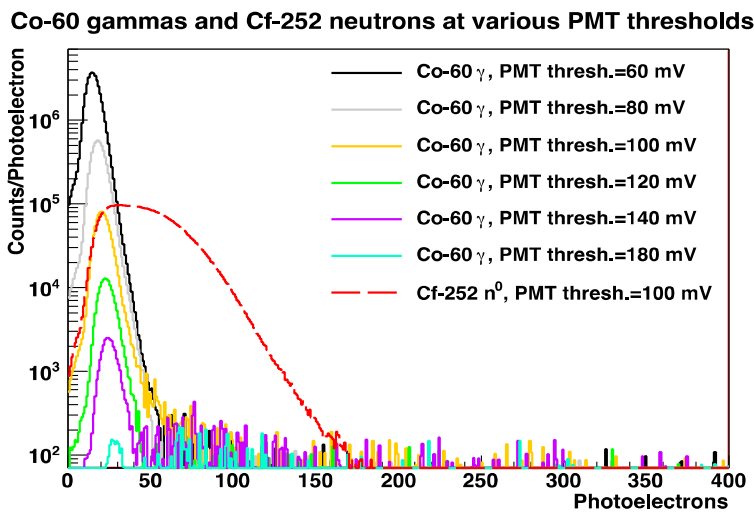

$\mathbf{1}$

4 the $100 \mathrm{mV}$ threshold. Note that the peak of this distribution is now at approximately 50 photoelectrons.

12 Note also that the background-subtracted ${ }^{60} \mathrm{Co}$ signal drops to near zero at a trigger threshold of $180 \mathrm{mV}$.

13 The best trigger level for the application will need to be adjusted as needed if background levels are high.

14 If the source to be investigated has low gamma ray activity the threshold can be set low in order to maximize neutron efficiency.

\section{6}

Figure 8: A series of one-hour ${ }^{60} \mathrm{Co}$ background subtracted runs with increasing trigger thresholds. Also shown for comparison is the neutron capture spectrum from a one-hour ${ }^{252} \mathrm{Cf}$ run at a high PMT threshold of $100 \mathrm{mV}$.

Figure 8 shows the spectral response as a function of PMT discriminator threshold for a set of one-hour background subtracted ${ }^{60} \mathrm{Co}$ data acquisitions. For reference, all the spectra shown previously were obtained with a relatively low nominal threshold setting of $60 \mathrm{mV}$ per PMT. Our motivation was to find a threshold setting that eliminates ${ }^{60} \mathrm{Co}$ events, and then to test its utility for fission sources that included an intense background gamma ray component. The ${ }^{60} \mathrm{Co}$ trigger rate at $100 \mathrm{mV}$ is approximately the same as the $60 \mathrm{mV}$ no-source trigger rate. We also show for reference the spectral response of the ${ }^{252} \mathrm{Cf}$ source at

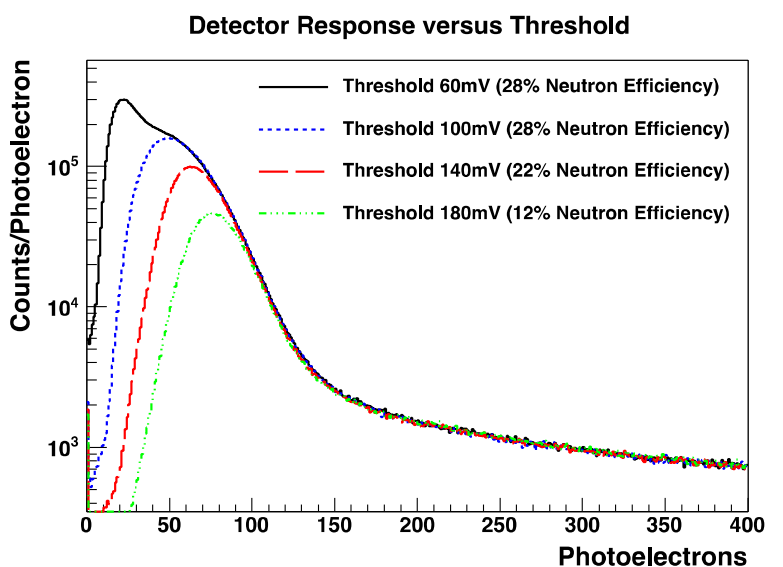

Figure 9: The spectral response of the detector to a 252Cf source for various PMT threshold settings. The resulting neutron detection efficiencies at each threshold are also shown. 
1

2 Figure 9 shows the detector response to ${ }^{252} \mathrm{Cf}$ as a function of the PMT threshold. Based on the trigger

3 threshold absolute efficiency of $28 \%$ defined earlier with the analysis threshold of 50 photoelectrons, the

4 absolute efficiencies at higher thresholds were calculated from the relative changes in the integrated

5 detection rate at each PMT threshold.

6

7

8

9

10

11

12

13

14

15

16

17

18

19

20

21

22

23

24

25

26

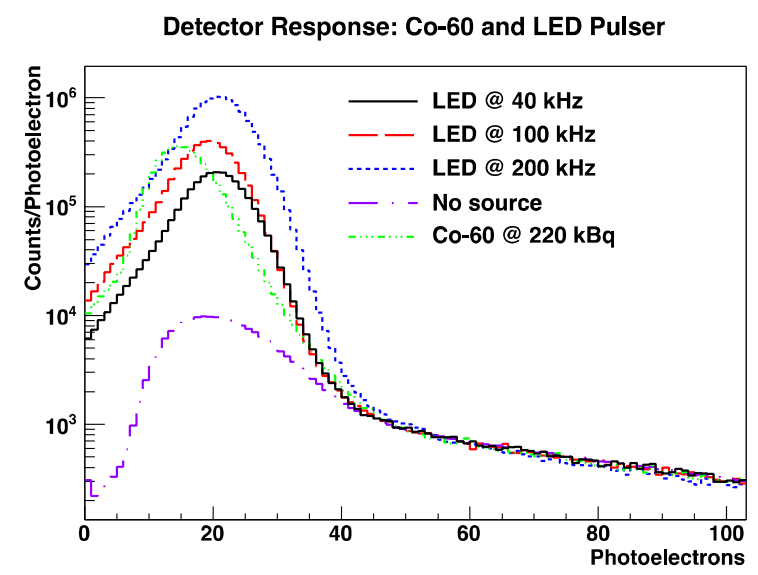

Figure 10: An investigation of the equivalence, in terms of detector response, of an LED pulser operating at different frequencies and a $220 \mathrm{kBq}{ }^{60} \mathrm{Co}$ source. The LED bias was set to 0.88 volts. For this bias, a $100 \mathrm{kHZ}$ LED rate models the energy output of a ${ }^{60} \mathrm{Co}$ source reasonably well, especially at the high-energy tail near 50 photoelectrons.

The question of neutron efficiency and detector response as a function of very high levels of low energy gamma ray background is a very important one for any technique in this field. In principle, the Cherenkov light output from gamma rays less than about $1 \mathrm{MeV}$ should be small, as the resulting Compton scattered electrons are generally only slightly above the Cherenkov threshold. The use of both fresh and spent fuel samples was outside the scope of this study. However, we experimentally modeled the presence of a

19 fission source with an intense gamma ray component by a combining multiple sources as proxies for a high 20 activity gamma ray source - a low intensity ${ }^{60} \mathrm{Co}$ source, a green LED mounted inside the detector, and the $211 \mu \mathrm{Ci}^{252} \mathrm{Cf}$ source. The first task was to find an LED bias voltage (i.e. light intensity per pulse) that closely

22 matches the detector response obtained from the ${ }^{60} \mathrm{Co}$ source. Figure $\mathbf{1 0}$ shows the detector response

23 obtained using the optimum bias in our case (60 nanosecond pulses at 0.88 volts). The ${ }^{60}$ Co source at 220

$24 \mathrm{kBq}$ provides a trigger rate and energy spectrum approximately similar to the LED pulsing at $100 \mathrm{kHz}$. In

25 similar fashion to the detector response to ${ }^{60} \mathrm{Co}$, the upper edge of the LED spectrum falls off at

26 approximately 50 photoelectrons. 

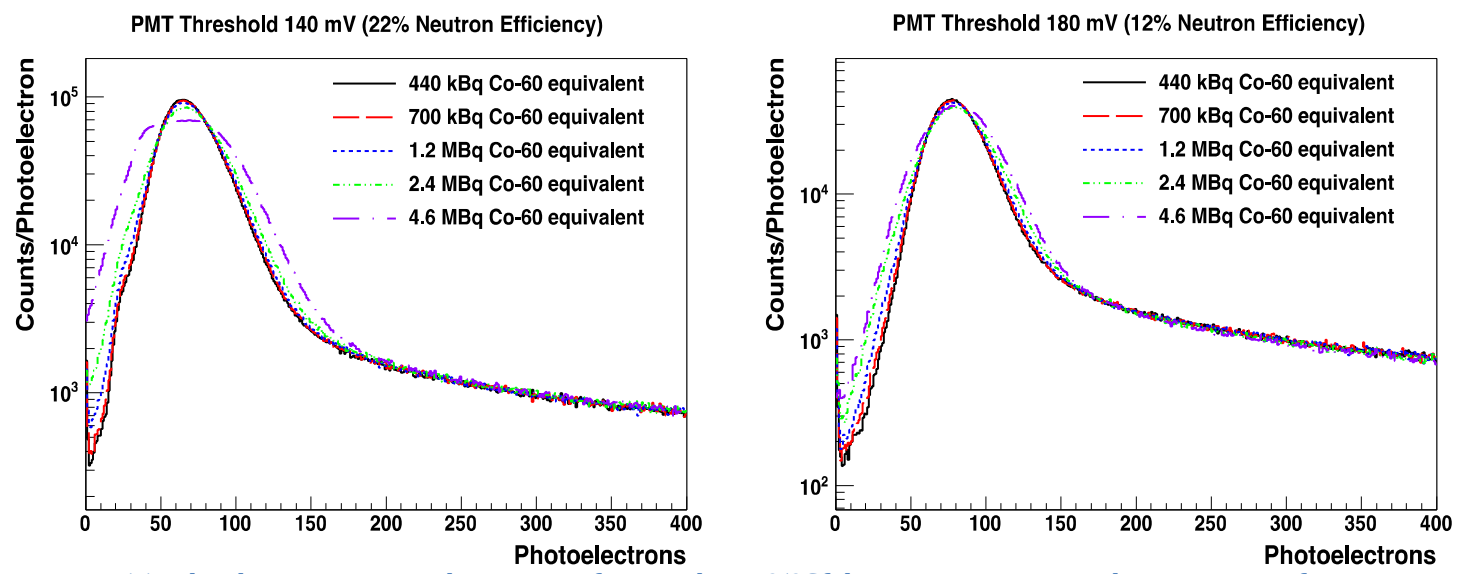

Figure 11: The detector spectral response for one-hour ${ }^{252} \mathrm{Cf}$ data acquisitions in the presence of progressively more intense low energy background. The ${ }^{60} \mathrm{Co}$ equivalent rates were modeled by combining a ${ }^{60} \mathrm{Co}$ source and a pulsing LED. We show the effect for two different PMT trigger thresholds, $140 \mathrm{mV}$ and $180 \mathrm{mV}$, corresponding to $22 \%$ and $12 \%$ neutron efficiency respectively.

7

Figure 11 shows the detector spectral response to the $1.0 \mu \mathrm{Ci}{ }^{252} \mathrm{Cf}$ source as a function of different

low energy background intensities. The ${ }^{60} \mathrm{Co}$ and LED related backgrounds were all below threshold, however, even when low energy backgrounds are not energetic enough to trigger the detector on an event-by-event basis, pileup may degrade the energy resolution of the detector at higher energies.

This pileup may arise when the rate of low energy gamma-rays is so high as to create a small amplitude, but nearly constant 'wash' of Cherenkov light, superimposed on the Cherenkov light created by real neutron captures. Recall that at low background intensities, an analysis cut at 50 photoelectrons rejects nearly all of the ${ }^{60} \mathrm{Co}$ gamma rays. At high intensities however, we reject these events with the trigger so as not to saturate the DAQ. We tested two high PMT threshold settings $140 \mathrm{mV}$ and $180 \mathrm{mV}$, corresponding to neutron efficiencies of $22 \%$ and $12 \%$ respectively. The various high intensity backgrounds were modeled by varying the LED rate in combination with the fixed ${ }^{60} \mathrm{Co}$ source. The ${ }^{60} \mathrm{Co}$ equivalent rates were calculated assuming the conversion calculated above (100 $\mathrm{kHz}$ LED $\approx 220 \mathrm{kBq}{ }^{60} \mathrm{Co}$ ). We observed reasonably consistent detector response (resolution and efficiency) at ${ }^{60} \mathrm{Co}$ equivalent background levels up to $\sim 4 \mathrm{MBq}$. If, as before, we accept events between 50 and 200 photoelectrons as neutron candidates, the neutron count rate at each background level is given in Table 1. The data indicate that neutron detection efficiency is consistent to within $5 \%$ up to a ${ }^{60} \mathrm{Co}$ equivalent source intensity of $\sim 4 \mathrm{MBq}$. Note also that the lower threshold 
1 (140 mV), capable of $22 \%$ neutron efficiency, is as effective at providing neutron detection

2 consistency over a large range of background intensities as the higher threshold setting (180 $\mathrm{mV})$.

3

$4 \quad$ Table 1: Measured neutron detection rate for steadily increasing rates of ${ }^{60} \mathrm{Co}$ equivalent background source 5 intensity.

\begin{tabular}{|l|l|l|}
\hline \multirow{2}{*}{$\begin{array}{l}{ }^{60} \text { Co Equivalent } \\
\text { Background Rate }\end{array}$} & \multicolumn{2}{|l|}{ Neutron Detection Count rates (Hz) } \\
\cline { 2 - 3 } & $\begin{array}{l}140 \mathrm{mV} \text { threshold } \\
(22 \% \text { neutron } \\
\text { efficiency) }\end{array}$ & $\begin{array}{l}180 \mathrm{mV} \text { threshold } \\
(12 \% \text { neutron } \\
\text { efficiency) }\end{array}$ \\
\hline $440 \mathrm{kBq}$ & $965 \mathrm{~Hz}$ & $478 \mathrm{~Hz}$ \\
\hline $700 \mathrm{kBq}$ & $965 \mathrm{~Hz}$ & $480 \mathrm{~Hz}$ \\
\hline $1.2 \mathrm{MBq}$ & $964 \mathrm{~Hz}$ & $485 \mathrm{~Hz}$ \\
\hline $2.4 \mathrm{MBq}$ & $968 \mathrm{~Hz}$ & $493 \mathrm{~Hz}$ \\
\hline $4.6 \mathrm{MBq}$ & $1010 \mathrm{~Hz}$ & $558 \mathrm{~Hz}$ \\
\hline
\end{tabular}

6

\section{5. Discussion and Conclusions}

9 In the following we evaluate the performance of the WBWC relative to existing ${ }^{3} \mathrm{He}$ techniques using the

10 safeguards figure-of-merit (FOM) defined earlier for two scenarios, the NDA of a fresh fuel SNM sample

11 without a significant high-energy gamma ray emission rate and the NDA of a spent fuel sample with a high

12 gamma ray emission rate. For fresh fuel samples with low rates of gamma ray emission, the most

13 significant backgrounds come from high-energy gamma rays incident on the detector from the local

14 environment and high-energy cosmogenic muons and gamma rays. For this detector, we select neutron

15 capture events on the basis of the detector response, between 50 and 200 photoelectrons. We have shown

16 that the counter has a neutron detection efficiency of $28 \%$ with this selection criterion. The neutron capture

17 time, or die-away time is $16 \mu$ s. The neutron selection criterion almost entirely eliminates low energy

18 gamma rays $(<\sim 2 \mathrm{MeV})$ incident on the detector, whether they be from the local environment, or from

19 radioactivity in the source under investigation (see Figure 4 and 6). The remaining non-source

20 backgrounds are primarily due to cosmic ray particles such as muons, neutrons and gammas, or neutrons

21 from the local environment. Fortunately these backgrounds can be measured very accurately given the

22 stable detector performance demonstrated over time periods of three months.

23

24 The safeguards FOM for the WBWC is 
$2 \quad F O M=\frac{28}{\sqrt{16}}=7.0$

3

4 This compares favorably with alternative systems such as the Boron plate detector of [18] $(\mathrm{FOM}=2.74)$,

5 and the ${ }^{3} \mathrm{He}$-based HLNCC-II detector, a safeguards standard employed by many nuclear facilities and the

6 IAEA [19] $(\mathrm{FOM}=2.67)$. The FOM here is higher primarily because of the efficiency is relatively high,

7 but also because the neutron capture time is short. The relatively high value of the FOM suggests that the

8 WBWC might the ideal detector technology in circumstances where source related background is in the

9 medium to low range.

10

11 For spent fuel samples, or any sample that emits very intense gamma ray backgrounds, a higher trigger

12 threshold might be utilized, so as to avoid digitizing too much background. As we have shown, the WBWC

13 was able to maintain a neutron efficiency of $22 \pm 1 \%$ in the presence of gamma ray backgrounds equivalent

14 to approximately $4 \mathrm{MBq}$ of ${ }^{60} \mathrm{Co}$. The FOM for intense gamma ray sources such as these is nevertheless

15 still very competitive - 5.5. For water Cherenkov systems, the level of background gamma ray

16 susceptibility is highly dependent on energy. Lower energy gamma rays, such as from ${ }^{137} \mathrm{Cs}$ produce

17 almost no response when compared to ${ }^{60} \mathrm{Co}$. This is because the Cherenkov process in water is very

18 nonlinear in the energy region between 0.5 and $1.5 \mathrm{MeV}$. A question arises as to how best to evaluate the

19 effectiveness of various detection techniques in a consistent way, accounting for realistic background

20 conditions. A water Cherenkov detector is likely to compare more favorably with detectors evaluated for

21 their susceptibility to background ${ }^{137} \mathrm{Cs}$ gamma rays, while less so for ${ }^{60} \mathrm{Co}$. Both comparisons are common

22 in the literature (e.g. [6][7]). For this reason future studies of this technology will focus less on background

23 proxies such as ${ }^{137} \mathrm{Cs},{ }^{60} \mathrm{Co}$ and pulsing LEDs, in favor of real uranium and plutonium samples, including

24 fresh and spent fuel.

25

26 In conclusion, the WBWC evaluated here has an absolute neutron detection efficiency of approximately

$2728 \%$ and a FOM of 7.0. The efficiency was confirmed to within $0.5 \%$ using two independent methods. The

28 counter has very limited susceptibility to low energy gamma ray backgrounds such as ${ }^{137} \mathrm{Cs}$ and ${ }^{60} \mathrm{Co}$. We 
1 demonstrated a ${ }^{60}$ Co rejection factor of $\sim 10^{8}$ to 1 , for activities $\leq 220 \mathrm{kBq}$. For sources with higher

2 background activities the WBWC demonstrated consistent neutron detection efficiencies of $22 \pm 1 \%$ (FOM

3 5.5), up to ${ }^{60} \mathrm{Co}$-like source activities of approaching $4 \mathrm{MBq}$. In the near future we hope to be able to test

4 the WBWC with real world fission sources such as uranium and plutonium samples.

5

\section{\begin{tabular}{l|l}
6 & 6. Acknowledgements
\end{tabular}}

7 We would like to thank Darrell Carter for his expert technical help with both the detector design and

8 construction and John Steele for programming the trigger logic in the FPGA. This work was performed

9 under the auspices of the US Department of Energy by Lawrence Livermore National Laboratory under

10 Contract DE-AC52-07NA27344, release number LLNL-JRNL-654196. The authors wish to thank the DOE

11 NA-22 for their support of this project.

12

[1] R. T. Kouzes, "The 3He Supply Problem", PNNL-18388, (2009)

[2] T. M. Persons and G. Aloise, "Neutron detectors: Alternatives to using helium-3", Government

Accountability Office Technology Assessment, Report to Congress, (2011)

[3] D. A. Shea, D. Morgan, "The Helium-3 Shortage: Supply, Demand and Options for Congress", Congressional Research Service, (2010)

[4] A. Prosdocimi, "Non-destructive assay of fissile materials by detection and multiplicity analysis of spontaneous neutrons" nuclear science and technology, 1979.

[5] N. Ensslin, M. Krick, M. Pickrell, D. Reilly, J. Stewart "Chapter 6. Passive Neutron Multiplicity

Counting", Passive Nondestructive Assay of Nuclear Materials - 2007 Addendum,

Los Alamos National Laboratory Report, LA-UR-07-1402 (2007)

[6] D. Henzlova, L.G.Evans, H.O.Menlove, M.T.Swinhoe, J.B.Marlow, "Experimental evaluation of a boron-lined parallel plate proportional counter for use in nuclear safeguards coincidence counting", Nucl.

Inst. and Meth. A. V697 (2013), P114

[7] A.P. Simpson, S. Jones, M.J. Clapham and S. A. McElhaney, "A Review of Neutron Detection

Technology Alternatives to Helium-3 for Safeguards Applications”, INMM 52 ${ }^{\text {nd }}$ Annual Meeting (2011)

[8] D. Reilly, N. Ensslin, H. Smith, Jr., and S. Kreiner, "Passive Nondestructive Assay of Nuclear Materials (PANDA)", Los Alamos National Laboratory Report, LA-UR-90-732.

[9] H.O. Menlove, R.J. Dickinson, I. Douglas, C. Orr, F.J.G. Rogers, G. Wells, R. Schenkel, G. Smith, A. Fattah, A. Ramalho, "Field Test and Calibration of Neutron Coincidence Counters for High-Mass Plutonium Samples", Los Alamos National Laboratory Report, LA-10815-MS.

[10] S. Dazeley, A. Bernstein, N.S. Bowden, R. Svoboda, "Observation of neutrons with a Gadolinium doped water Cherenkov Detector", Nucl. Inst. and Meth.,A, V607 (2009), P616

[11] M. Sweany, A. Bernstein, N.S. Bowden, S. Dazeley, G. Keefer, R. Svoboda, M. Tripathi, "Large-scale gadolinium-doped water Cherenkov detector for nonproliferation", V654, (2011), P377

[12] S. Dazeley, M. Sweany, A. Bernstein, "SNM detection with an optimized water Cherenkov neutron detector", V693, (2012), P148

[13] W. Coleman, A. Bernstein, S. Dazeley, R. Svoboda, "Transparency of $0.2 \% \mathrm{GdCl} 3$ doped water in a stainless steel test environment”, Nucl. Inst. and Meth. A. V595 (2008), P339

[14] S. Agostinelli, et al., Nuclear Instruments and Methods A 506 (2003) 250.

[15] J. Allison, et al., IEEE Transactions on Nuclear Science NS-53 (1) (2006) 270.

[16] <http://Geant4.cern.ch>

[17] Y. Fukuda et al., "The Super-Kamiokande Detector", Nucl. Inst. and Meth.,A, V501 (2003), P418 
[18] D. Henzlova, H. O. Menlove, M. T. Swinhoe, J. B. Marlow, "Design and Development of a Safeguards Coincidence Counter Based on Boron-lined Proportional Detector Technology - High Level Neutron

counter Boron (HLNB)", Los Alamos National Laboratory Report, LA-UR-12-26261.

[19] Menlove H.O., Swansen J.E.; A high-performance neutron time correlation counter", Nuclear Technology 71, 497, (1985) 
FIGURE 1A

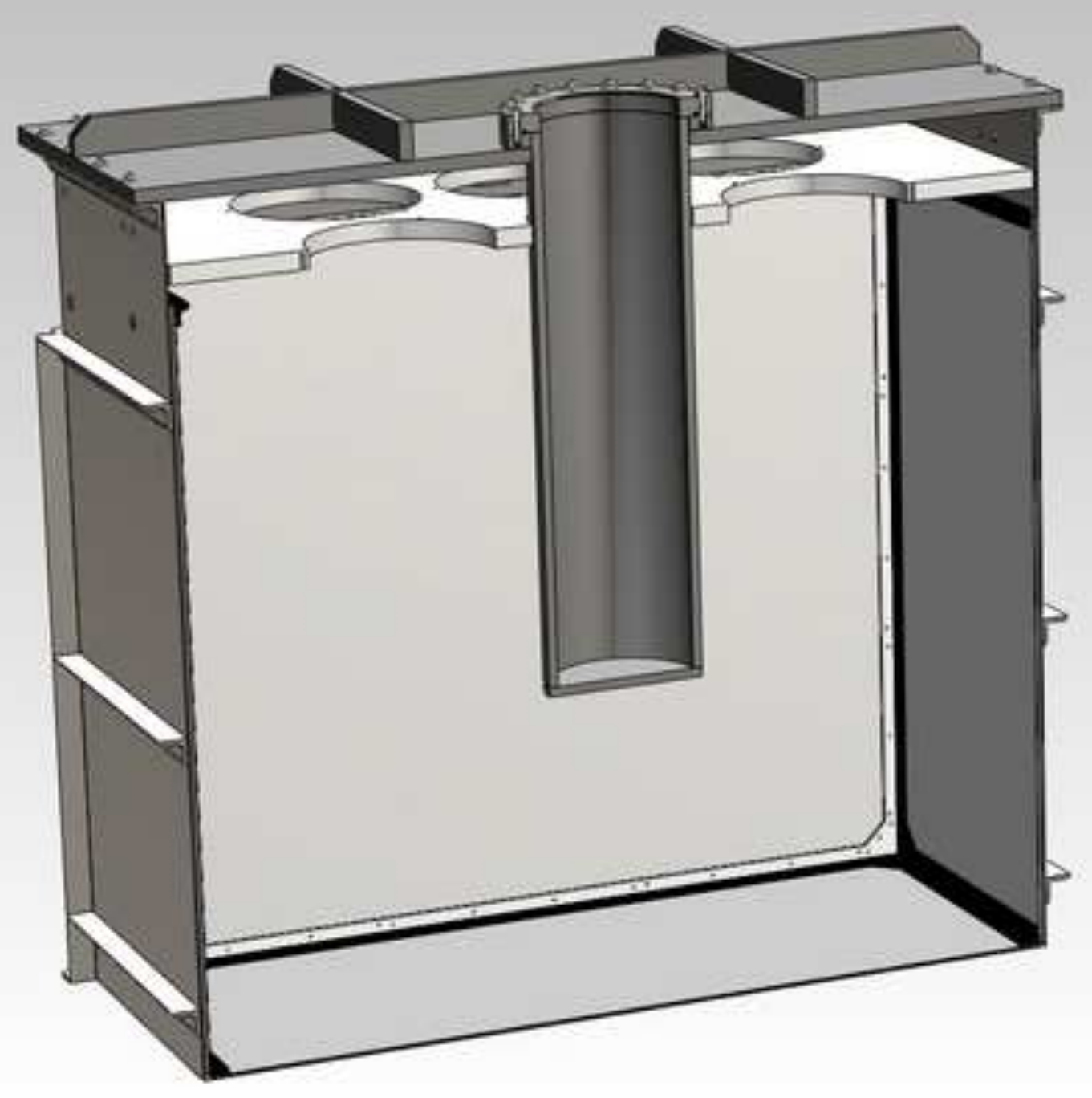


FIGURE 1A

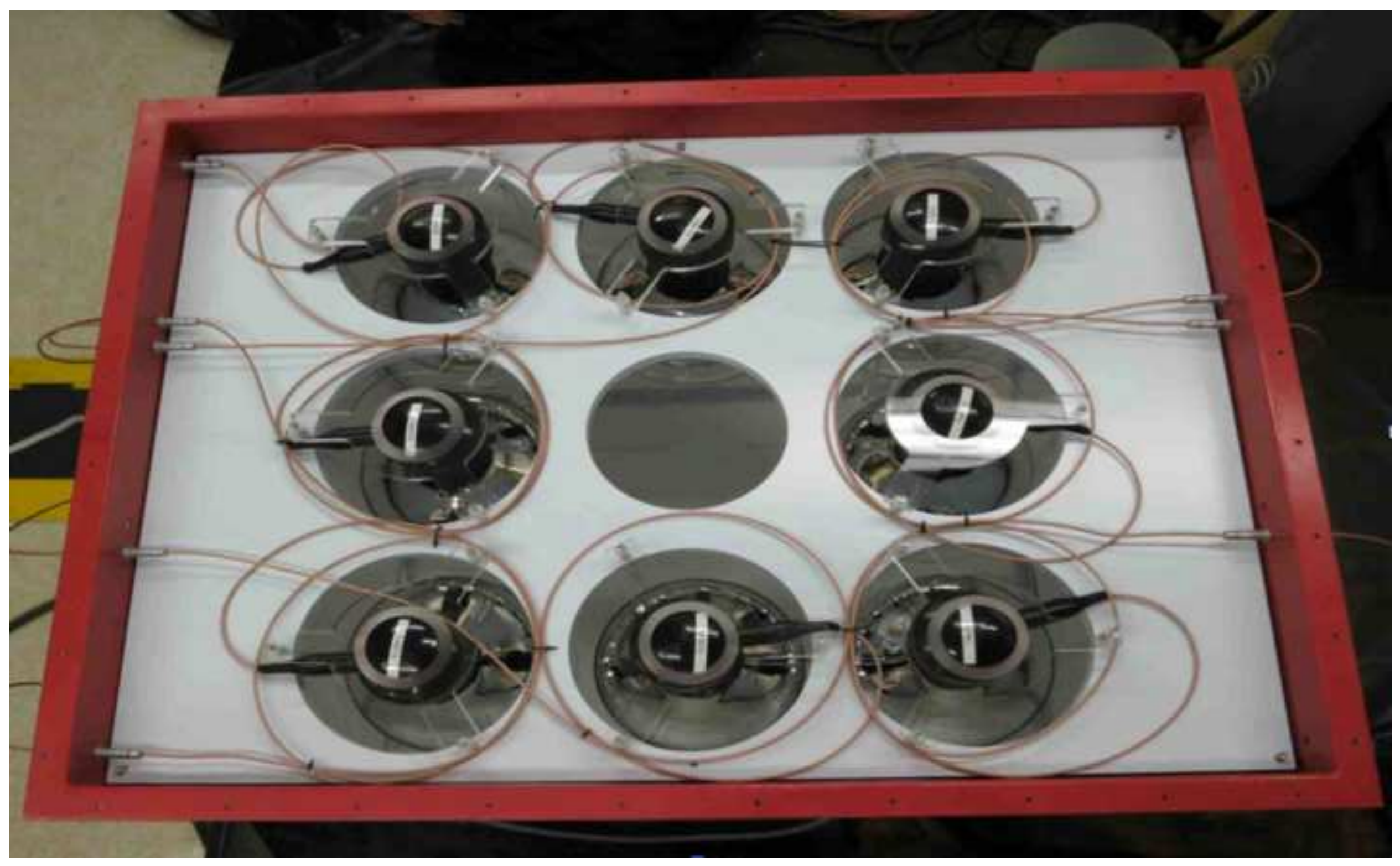




\section{Inter-event time}

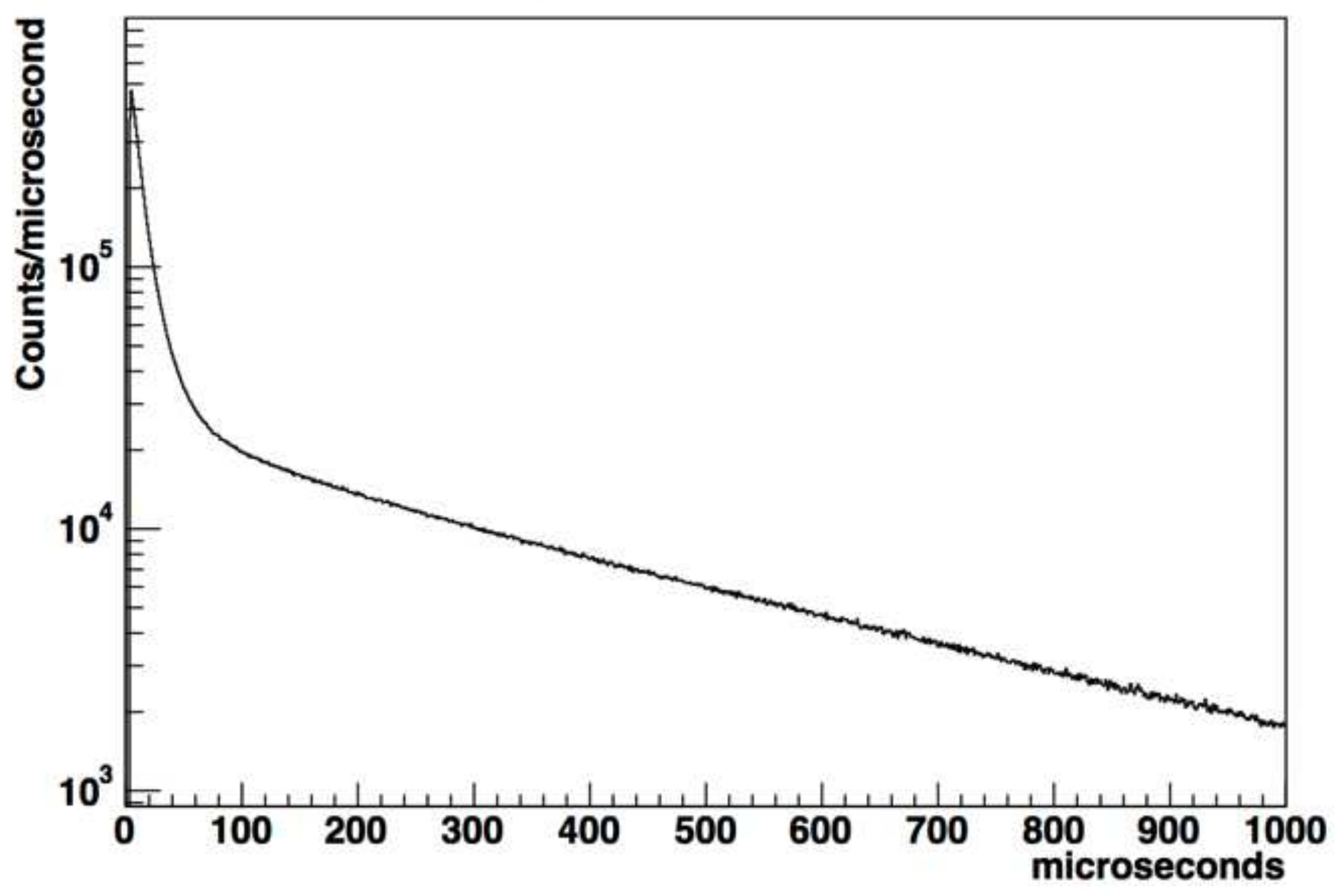


FIGURE 4

\section{0 kBq Co-60 Detector Response}

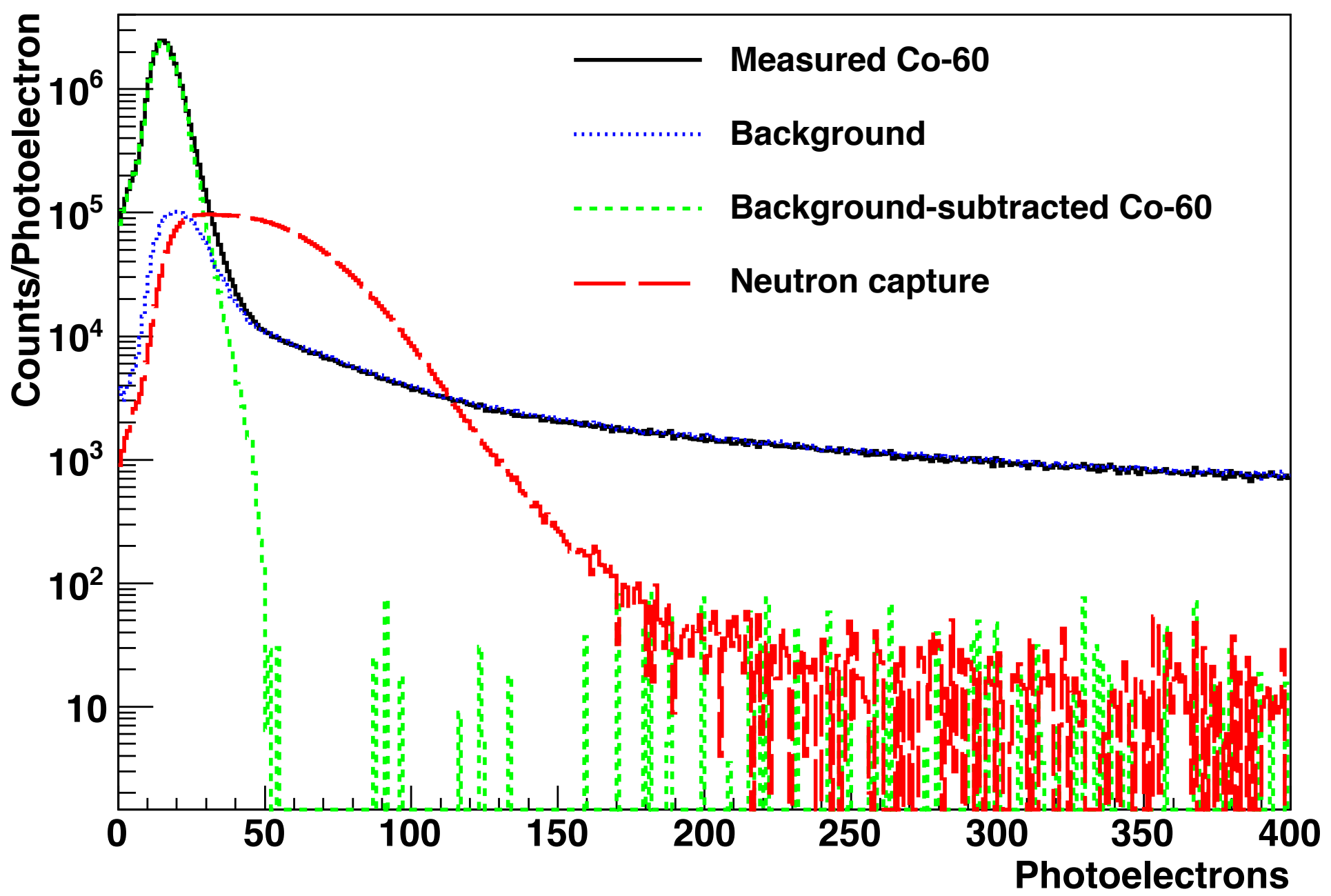


FIGURE 5

Neutron Capture Detector Response

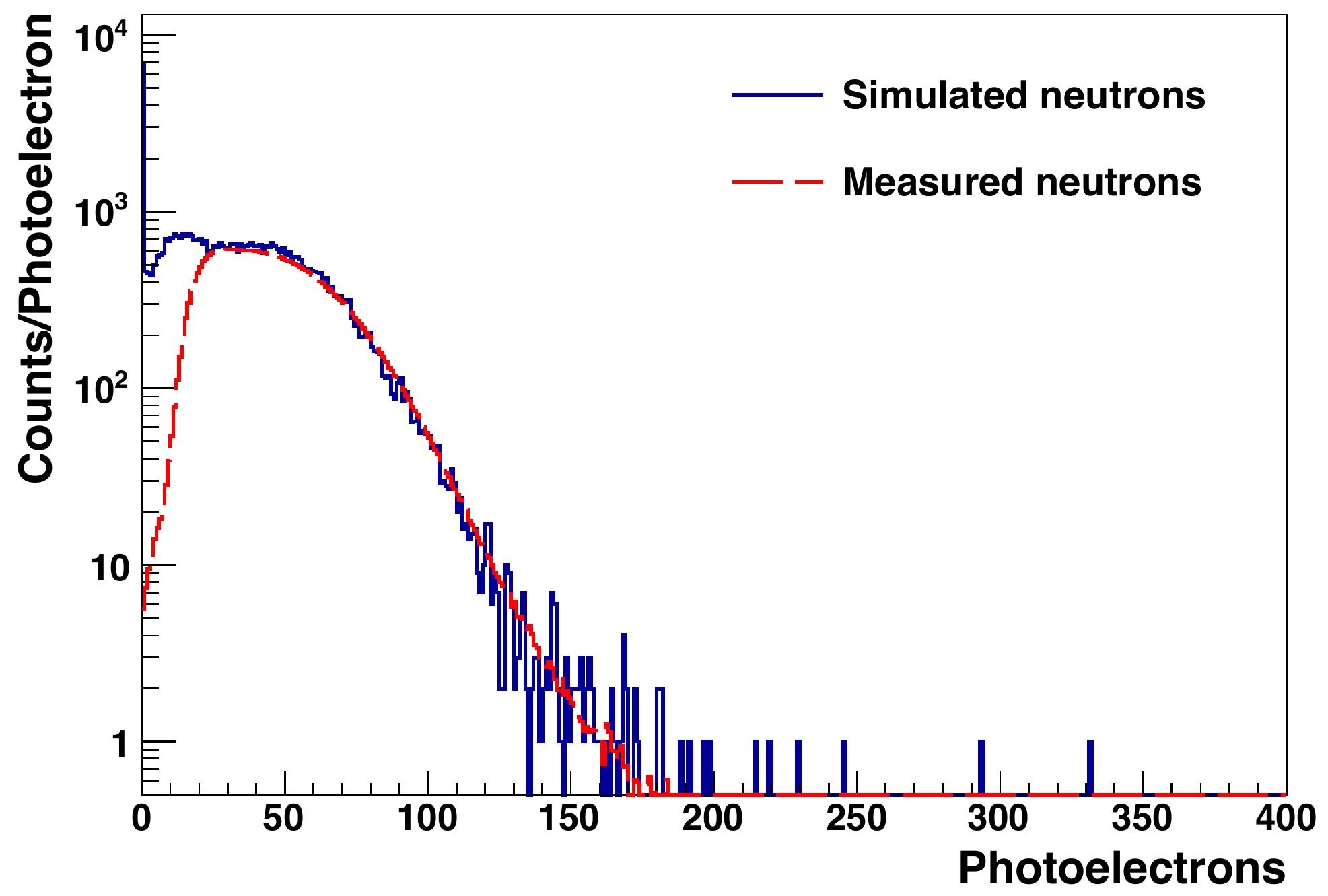


FIGURE 6A

\section{Spent Fuel Gamma ray Emission}

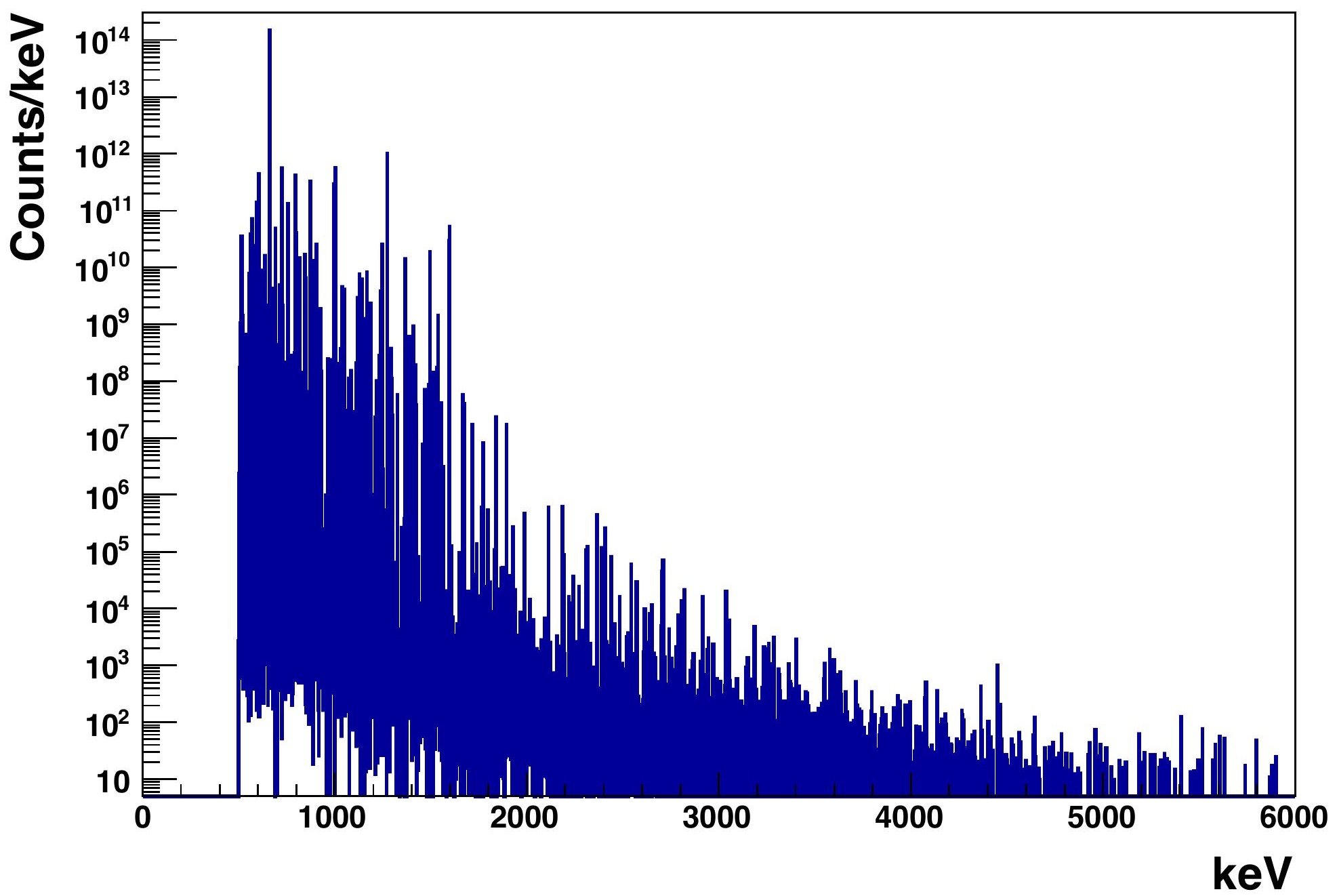


FIGURE 6B

\section{Detector Response}

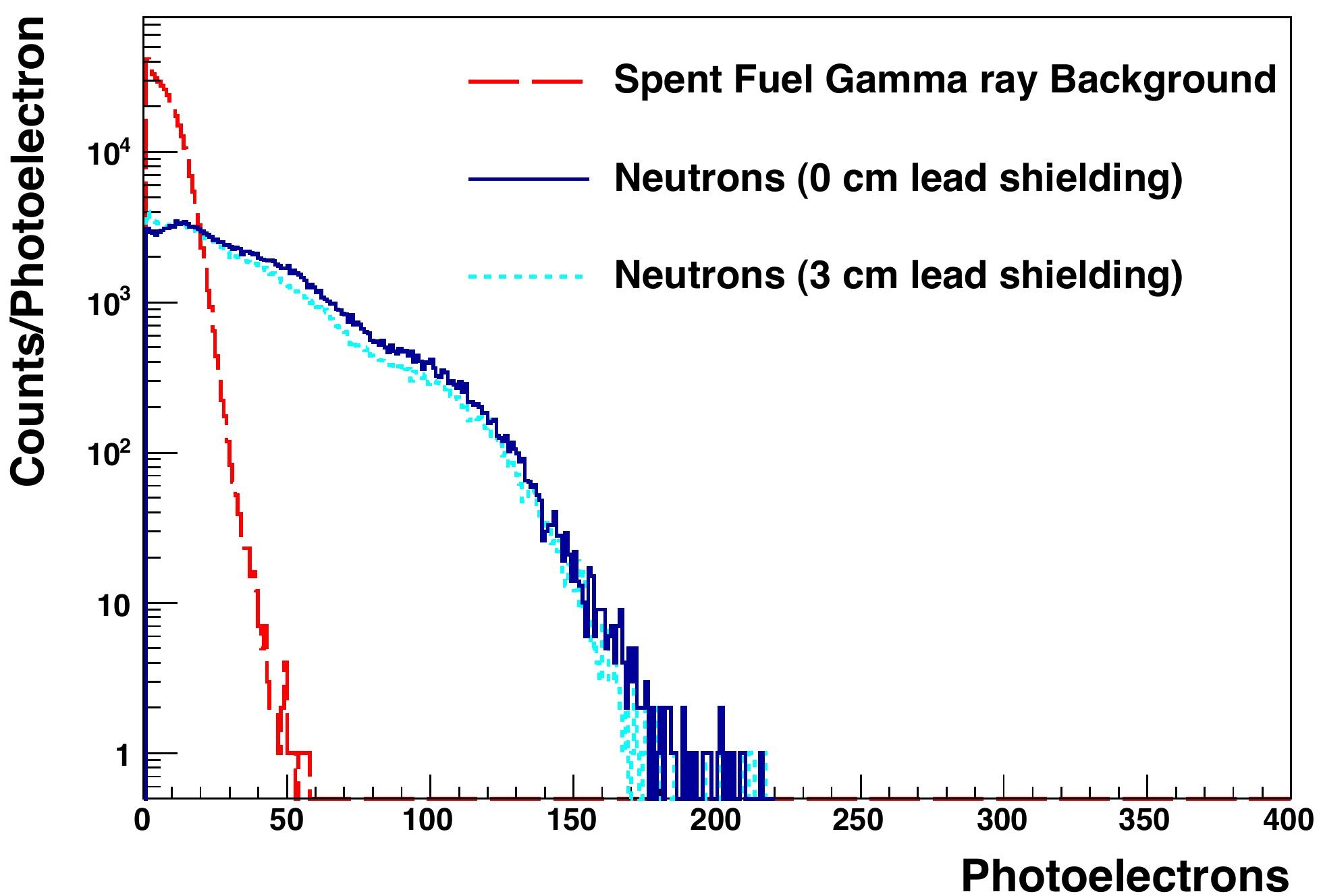


FIGURE 8

Co-60 gammas and Cf-252 neutrons at various PMT thresholds

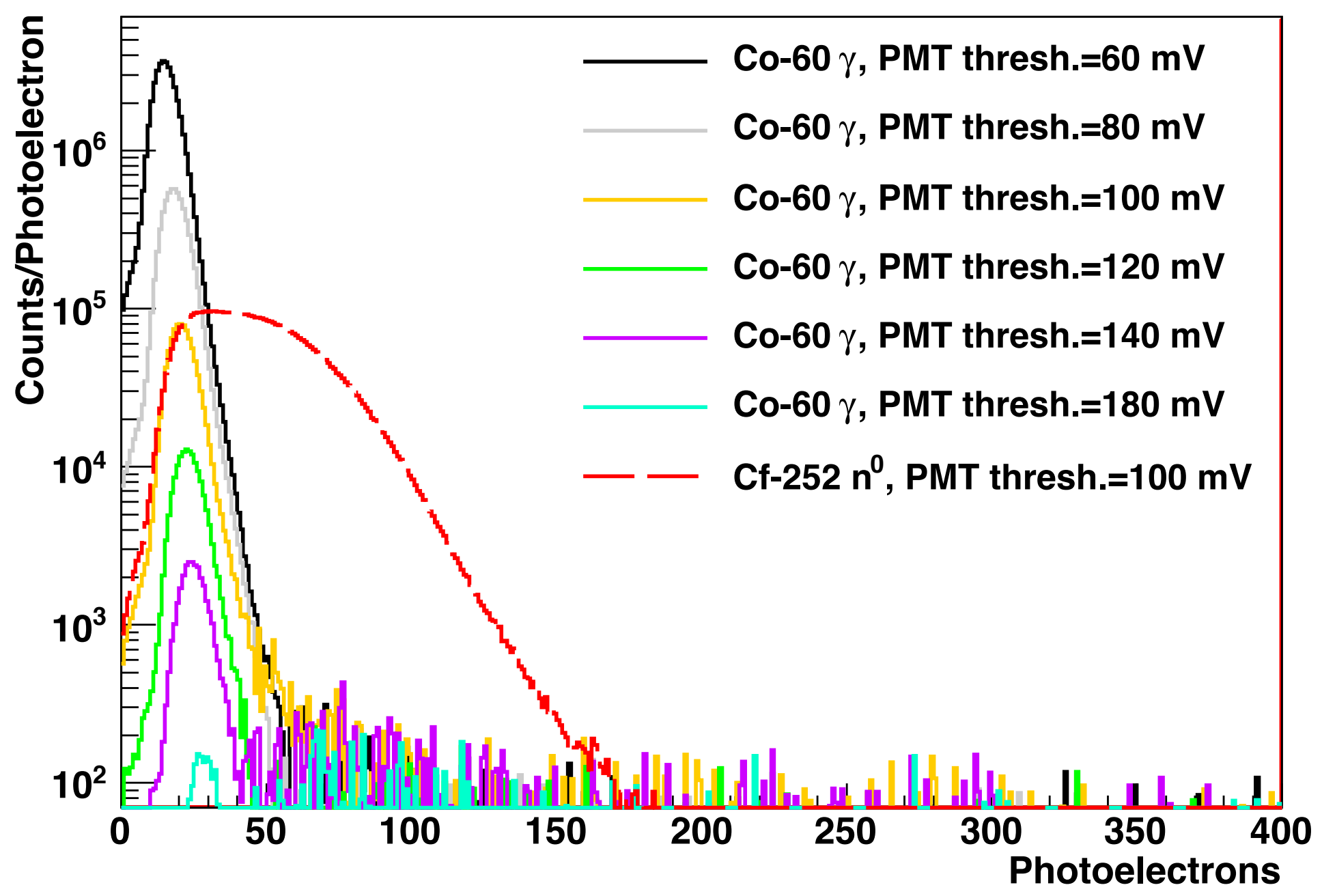


FIGURE 9

Detector Response versus Threshold

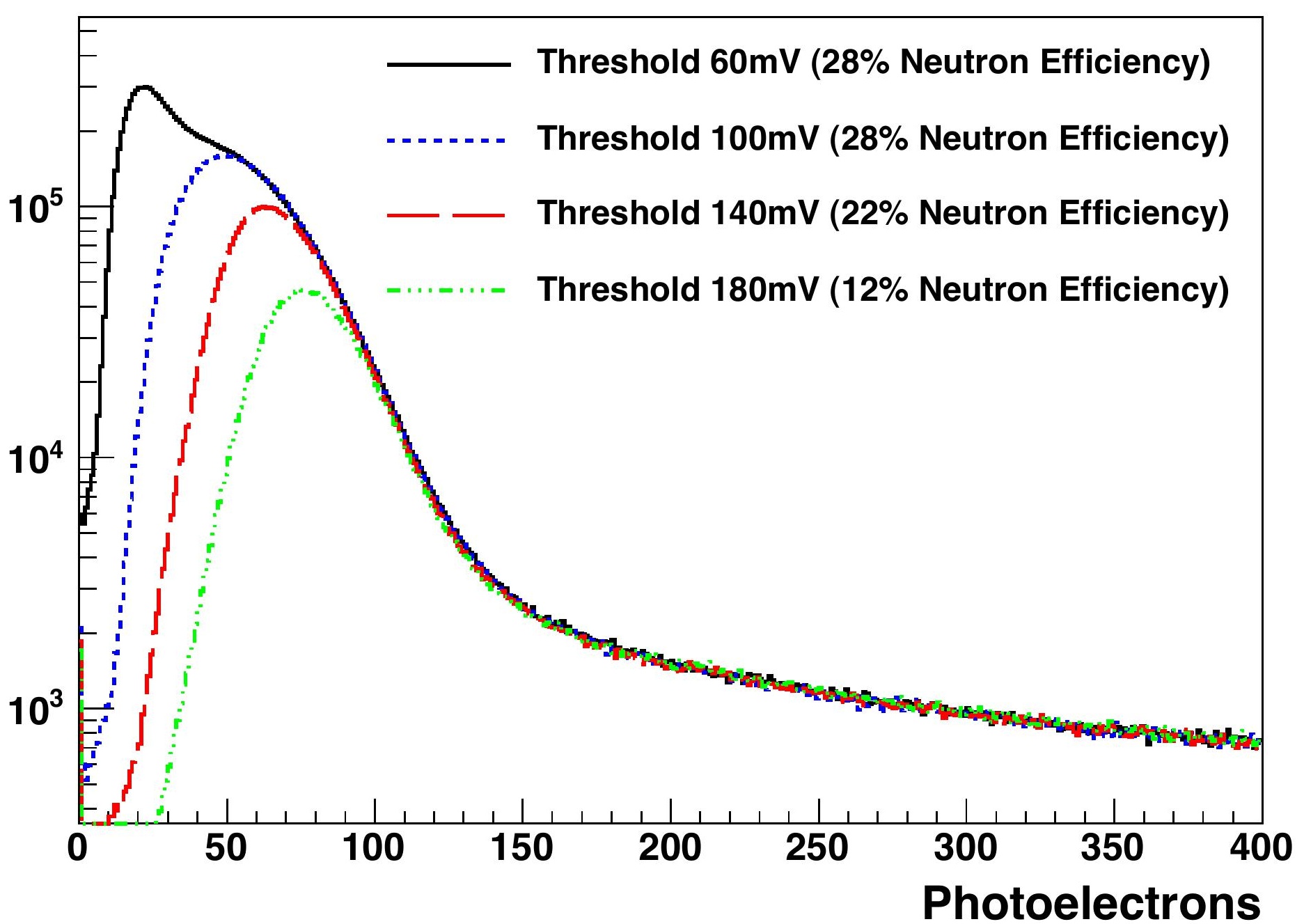


FIGURE 10

\section{Detector Response: Co-60 and LED Pulser}

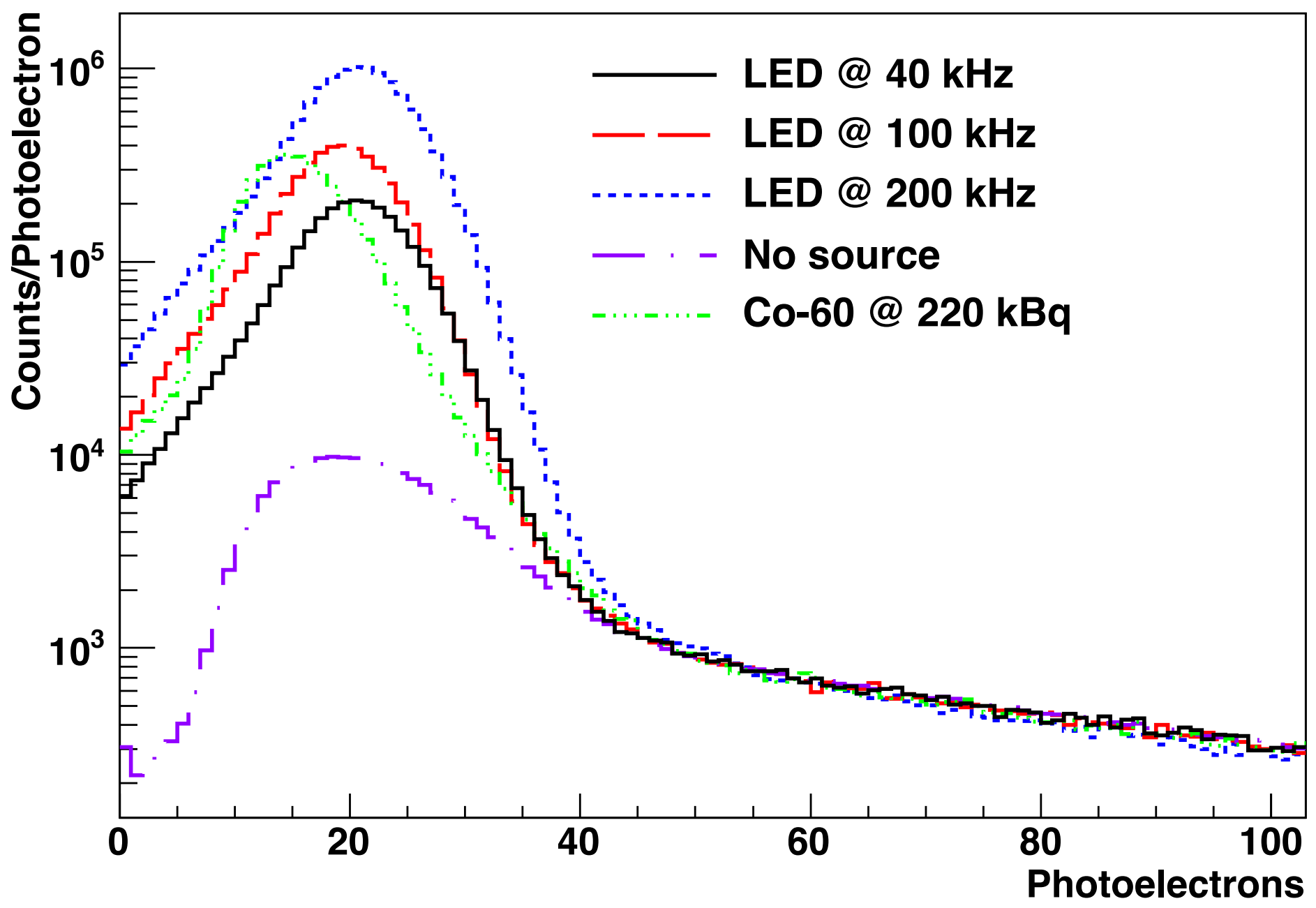


FIGURE 11A

PMT Threshold 140 mV (22\% Neutron Efficiency)

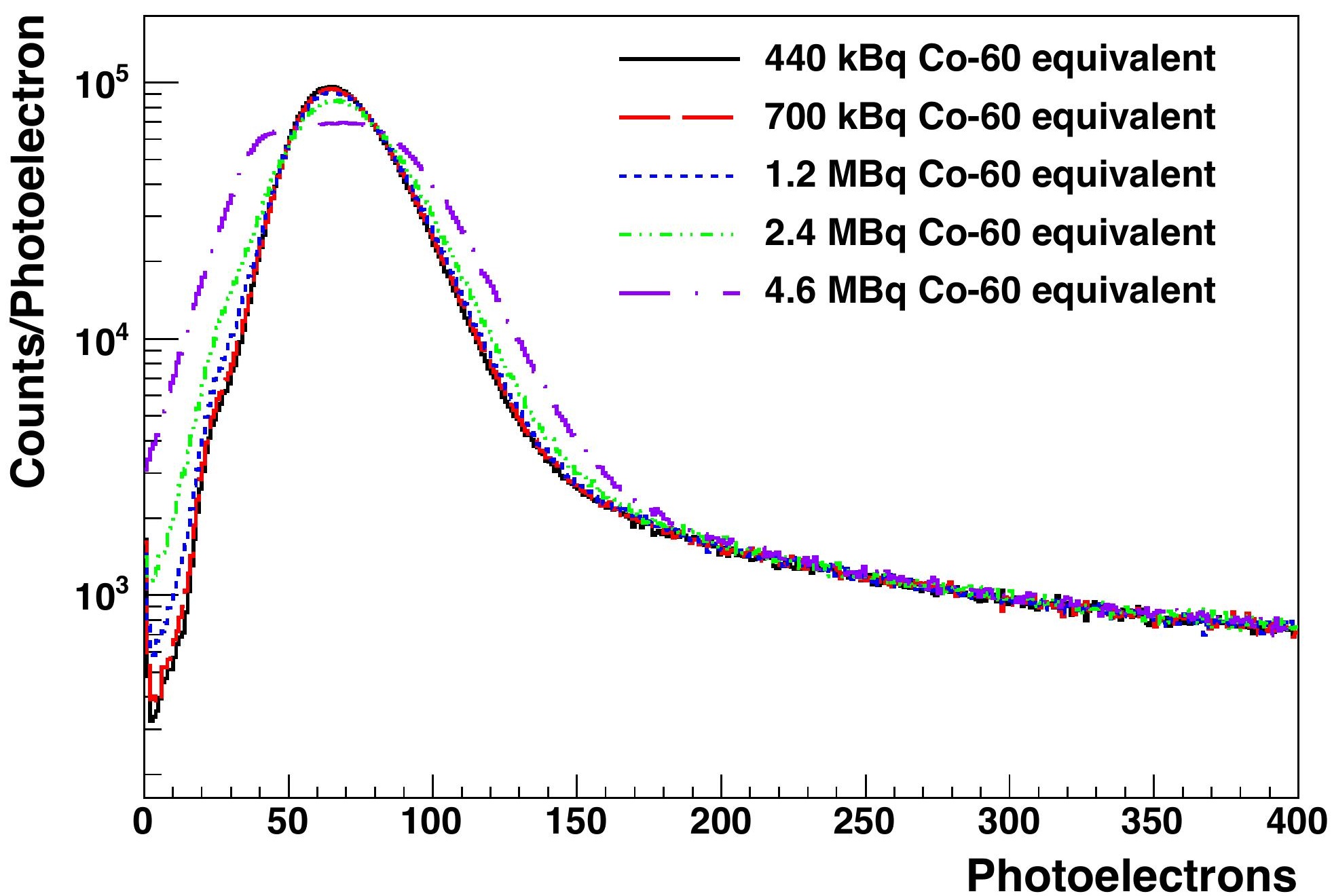


FIGURE 11B

PMT Threshold $180 \mathrm{mV}$ (12\% Neutron Efficiency)

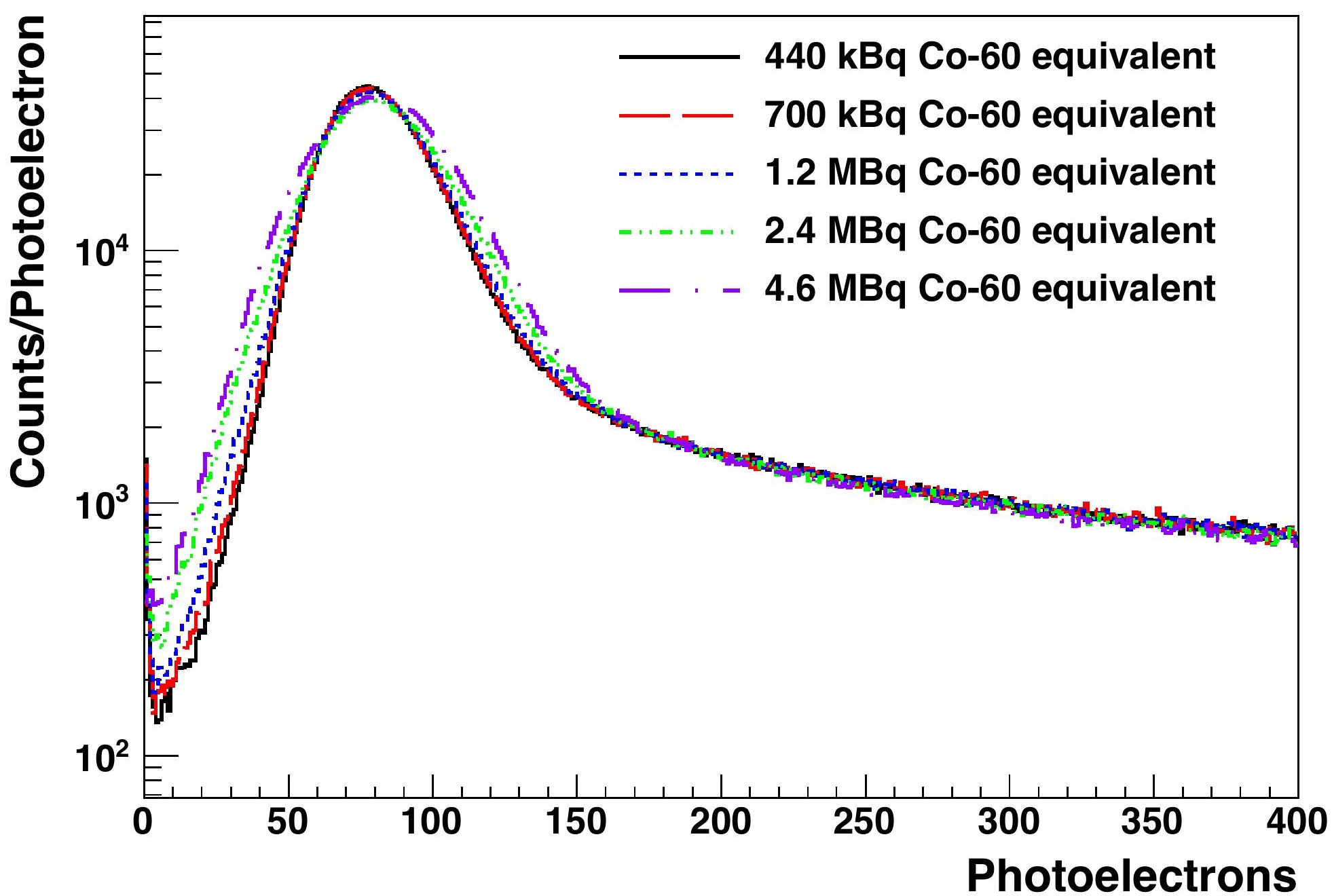


Cs-137 (330 kBq)

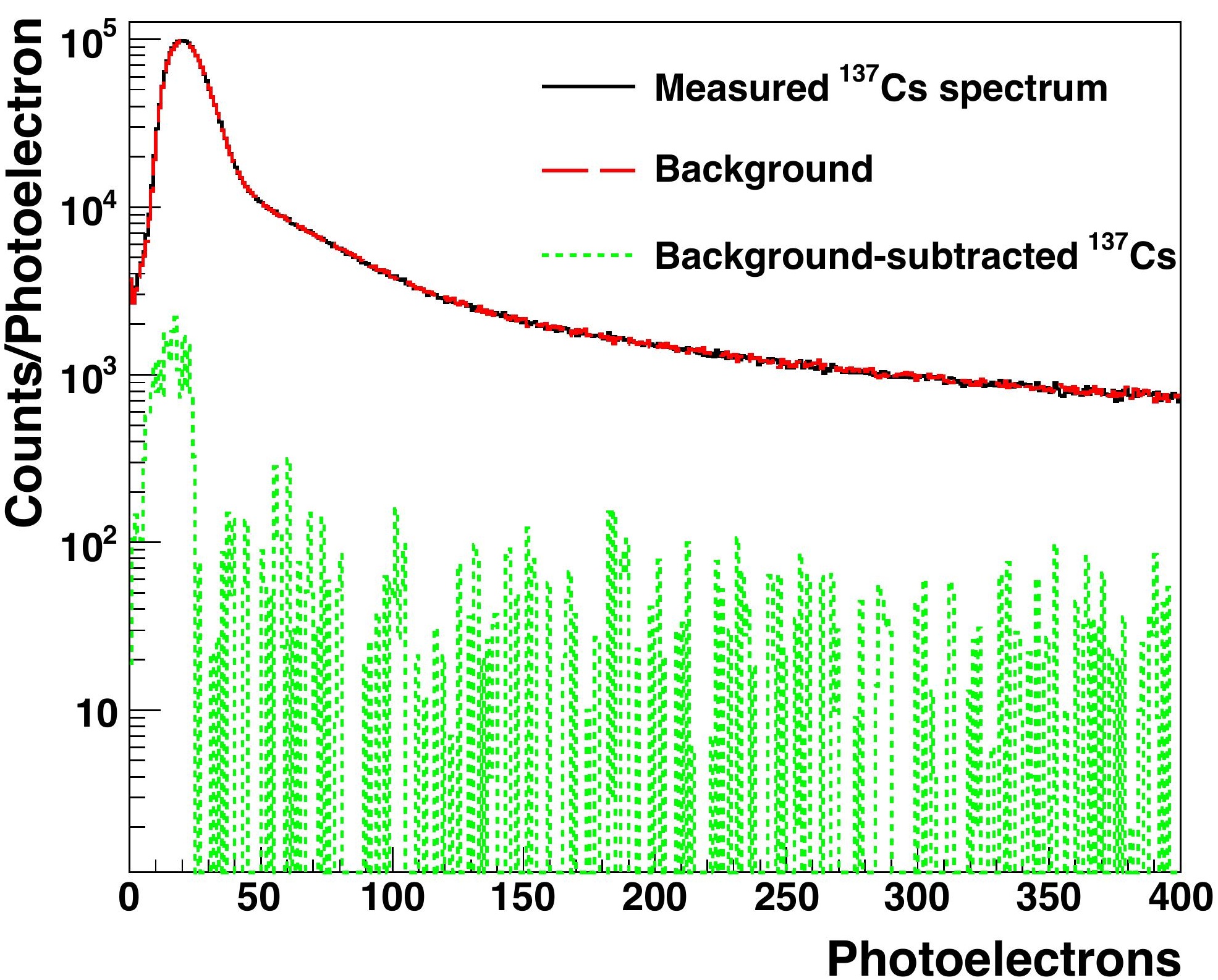


